\title{
Knowledge, Perceived Risk and Utilization of Prostate Cancer Screening Services among Men in Dar Es Salaam, Tanzania
}

\author{
Fidelis Charles Bugoye $\mathbb{D D}^{1}{ }^{1}$ Germana Henry Leyna, ${ }^{2}$ Kåre Moen, ${ }^{3}$ and Elia John Mmbaga \\ ${ }^{1}$ Department of Forensic Science and DNA Services, Government Chemist Laboratory Authority, Dar es Salaam, Tanzania \\ ${ }^{2}$ Department of Epidemiology and Biostatistics, Muhimbili University of Health and Allied Sciences, Dar es Salaam, Tanzania \\ ${ }^{3}$ Department of Community Medicine and Global Health, University of Oslo, Norway
}

Correspondence should be addressed to Fidelis Charles Bugoye; bugoye81@yahoo.co.uk

Received 28 May 2019; Revised 26 August 2019; Accepted 3 September 2019; Published 3 December 2019

Academic Editor: Hendrik Van Poppel

Copyright ( 2019 Fidelis Charles Bugoye et al. This is an open access article distributed under the Creative Commons Attribution License, which permits unrestricted use, distribution, and reproduction in any medium, provided the original work is properly cited.

\begin{abstract}
Background. Late diagnosis of prostate cancer is common in low and middle income countries and contributes to high morbidity and mortality of the disease. Utilization of prostate cancer screening services plays a major role in prevention of adverse outcomes. However, there is limited information on the knowledge about, the perceived risk of, and the utilization of prostate cancer screening in Tanzania. Objective. To determine knowledge and perceived risk of prostate cancer, and the utilization of prostate cancer screening services, and associated factors, among men in Dar es Salaam, Tanzania. Design. A population-based cross-sectional study involving men aged 40 years and above living in Dar es Salaam was conducted between May and August, 2018. Methodology. Participants were recruited through multistage random sampling and took part in structured face-to-face interviews. Categorical variables were summarized using proportions while continuous variables were summarized as medians and inter-quarterly range (IQR). Chi square test was used to compare differences between proportions, and logistic regression modelling was used to determine factors associated with utilization of prostate cancer screening. Both crude and adjusted odds ratios (OR), with corresponding 95\% confidence intervals, are reported. All analyses were two-tailed and the significance level set at 5\%. Results. A total of 388 men with a median age of 53 years (IQR 44-55) participated. Half (52.1\%) had poor knowledge about prostate cancer and prostate cancer screening. A third $(32.3 \%, n=125)$ perceived the risk of prostate cancer to be low. Only 30 respondents $(7.7 \%)$ had ever been screened for prostate cancer. Utilization of prostate cancer screening services was independently associated with age above 60 years $[\mathrm{AOR}=21.46,95 \% \mathrm{CI}: 6.23,73.93]$, monthly income above 305 US Dollars [AOR $=15.68,95 \%$ CI: 4.60, 53.48], the perceived risk of prostate cancer $[\mathrm{AOR}=16.34,95 \% \mathrm{CI}: 7.82,14.92]$ and knowledge about prostate cancer [AOR $=67.71,95 \%$ CI: 8.20, 559.57]. Conclusions. Knowledge about prostate cancer and prostate cancer screening services was low among men in Dar es Salaam with a third perceiving themselves to be at no risk for the disease. Utilization of screening services was low and associated with low income, younger age, low perceived risk of prostate cancer and low knowledge about the disease. Intervention measures aiming to increase knowledge about prostate cancer and screening services, and affordable provision of services, are urgently called for.
\end{abstract}

\section{Introduction}

Breast cancer in women and prostate cancer in men have now become the most commonly diagnosed cancers in many SubSaharan African countries. Prostate cancer is an adenocarcinoma of the male prostate and is one of the most common cancers in the world and a leading cause of cancer related deaths among men globally [1-3]. While prostate cancer is thus an important health burden among men across the globe, the highest incidence rates are found in sub-Saharan Africa
$[1,2,4,5]$. A systematic review of prostate cancer in Africa estimated an overall continent-wide pooled incidence rate of 21.95/100,000 population [2]. The Tanzania Cancer Registry indicates that prostate cancer is the most common cancer among men in Tanzania (followed by Karposi's sarcoma) with an incidence of 3,434 cases per year in 2012 [6].

While screening for prostate cancer using the prostate-specific antigen blood test (PSA) and digital rectal examination (DRE) are effective available measures for early detection of disease, utilization of these services ranges from $0 \%$ to $15 \%$ in 
Africa [4, 7-10]. A majority of patients with prostate cancer in Tanzania report to health facilities when the cancer is locally advanced or metastatic. A recent study reported that $21 \%$ of men attending routine transurethral prostatectomy were incidentally identified to have prostate carcinoma [11].

Various factors associated with utilization of prostate cancer screening have been documented both in developed and developing countries. A recent study reported that African and African American men were less likely than European and European American men to seek prostate cancer-screening as a direct or indirect consequence of financial barriers, lack of health insurance, and/or poor health-seeking behaviour [10]. Studies have also found that perceived risk of prostate cancer and low knowledge about the disease and prostate cancer screening methods play an important role in cancer screening utilization [7, 10, 12-15]. Good knowledge about and understanding of a disease is generally associated with a more optimal healthcare-seeking attitude and behaviour [9, $13,16]$.

Recently there have been a number of public campaigns to promote prostate cancer awareness in Tanzania, but the utilization of prostate cancer screening services has not been evaluated. The present study therefore aimed to examine the level of knowledge about prostate cancer, perceived risk of the disease, and utilization of prostate cancer screening services, and their associated factors, among men in Dar es Salaam in order to inform ongoing and new intervention measures.

\section{Population and Methods}

2.1. Study Design and Population. A population-based crosssectional study was conducted from May to August 2018 among men aged 40 years and above who were residents of Dar es Salaam (had an address in the city and had lived in it for more than one year). Based on the level of knowledge of prostate cancer and prostate cancer screening reported in a study from Kenya [17], sample size calculation indicated that recruitment of 384 study participants would yield $80 \%$ power at $5 \%$ error rate to estimate our outcome.

2.2. Sampling Procedure. Multistage cluster sampling was used to recruit participants for this study. We first randomly selected two of Dar es Salaam's five administrative municipalities (Kinondoni and Ubungo). From each of these, two wards were selected randomly (Sinza, Goba, Ndugumbi and Mwananyamala), from each of which two streets were randomly selected, from each of which two administrative cells were randomly selected. A list of households with eligible men was obtained from the ward office and served as our sampling frame within these administrative cells. Eligible participants were recruited through house to house visit during the evening.

2.3. Data Collection Tool. A questionnaire was followed during structured face-to-face interviews to collect data on socio-demographic characteristics of the respondents, their knowledge about and perceived risk of prostate cancer, and their utilization of screening services. A set of questions were used to measure knowledge of prostate cancer and pretested to assess their face validity prior to actual data collection. All questions were developed in English and then translated into Swahili, the language used during the interview.

2.4. Data Analysis. Data were entered into and analysed in the Statistical Packages for the Social Sciences (SPSS) version 20.0. Categorical variables were summarized using proportions while continuous variables were summarized as medians and inter-quarterly range (IQR). Knowledge (8 questions) and internal consistency was examined using Cronbach's alpha (0.856). Chi square test was used to compare differences between proportions and both bivariate and multivariate logistic regression modelling was used to determine independent factors influencing the utilization of prostate cancer screening services. Both crude odds ratios (OR) and adjusted odds ratios (AOR) were calculated and presented with corresponding 95\% confidence intervals. All analyses were two-tailed and the significance level set at $5 \%$.

2.5. Ethical Issues. Ethical approval for this research was obtained from MUHAS Ethical Research Committee (MU/ PGS/SAEC/VOL.IX/56) and the permission to conduct the study was thereafter requested from local authorities in Kinondoni and Ubungo municipalities. All study participants provided written informed consent prior to the interview. Confidentiality was maintained and no names were recorded in any questionnaire. Interviews were conducted in privacy.

\section{Results}

About 400 men were approached to consent and participate in the study-a total of $388(97 \%)$ men with a median age of 53 years (IQR: 44-55) living in Ubungo and Kindondoni municipality of Dar es Salaam participated in the study. Majority were married $(85.1 \%)$ and about a third had completed secondary education with only $1 \%$ reporting no formal education. Nearly half (45.6\%) were employed whereas $39.9 \%$ engaged in business and $10.8 \%$ were retired. The median monthly earning among respondents was 305 US Dollars (IQR $=174-348$ US Dollars) (Table 1).

\subsection{Knowledge about Prostate Cancer and Prostate Cancer} Screening Methods. Overall, 48\% $(n=186)$ of the study participants were knowledgable about prostate cancer and prostate cancer screening method (Figure 1).

Table 2 presents the proportion of responses to various knowledge questions. Knowing someone previously affected by prostate cancer, was reported by about half of the interviewees $(46.1 \%, n=179)$ while knowing someone currently was reported by $17.3 \%(n=67)$. The majority of participants $(93.3 \%, n=362)$ knew that prostate cancer is a disease that affects men, nearly two thirds $(63.7 \%, n=247)$ knew that the disease is severe, and half $(51.3 \%, n=199)$ knew that the disease is curable if detected early. While only $27.3 \%(n=106)$ had knowledge about the symptoms of prostate cancer, $62.4 \%$ $(n=242)$ knew about the screening methods (PSA and/or DRE). 
TABLE 1: Distribution of socio-economic and demographic characteristics of the study respondents $(n=388)$.

\begin{tabular}{lc}
\hline Variable & $n(\%)$ \\
\hline Median age (IQR) & $53(44 ; 55)$ \\
Area of residence & \\
Kinondoni & $194(50.0)$ \\
Ubungo & $194(50.0)$ \\
Marital status & \\
Married & $330(85.1)$ \\
Separated & $14(3.6)$ \\
Widowed & $17(4.4)$ \\
Single & $27(7.0)$ \\
Level of education & \\
No formal education & $12(3.1)$ \\
Primary education & $103(26.5)$ \\
Secondary education & $129(33.2)$ \\
Diploma & $54(13.9)$ \\
University & $90(23.2)$ \\
Current occupation & \\
Employed & $177(45.6)$ \\
Business & $155(39.9)$ \\
Retired & $42(10.8)$ \\
Not working & $5(1.3)$ \\
Agriculture & $9(2.3)$ \\
Median monthly earning in tzs (IQR) & $305(174-348)$ US Dollars \\
\hline
\end{tabular}

3.2. Participants Perceived Risk of Prostate Cancer. When participants were asked how they perceived the risk of prostate cancer to be among men in general, $24.6 \%(n=95)$ said men are at high risk, $62 \%(n=240)$ said they are at some risk and $23.4 \%(n=52)$ that they are at no risk at all. With regards to the interviewee's individual (personal) risk of prostate cancer, $13.7 \%$ ( $n=53)$ felt they were at high risk, half $(54.0 \%, n=209)$ thought they were at some risk whereas about a third $(32.3 \%, n=125)$ assumed that they were at no risk of developing prostate cancer (Figure 1).

\subsection{Utilization of Prostate Cancer Screening Services among}

Respondents. The proportion of study participants who had ever utilized prostate cancer screening services was only $7.7 \%(n=30)$. Participants who were above 60 years, who had a monthly income above 305 US Dollars, who were knowledgable about prostate cancer and who perceived themselves to be at risk of prostate cancer, were more likely to have utilized screening services in the bivariate analysis (Table 3).

\subsection{Independent Factors Associated with Utilization of Prostate}

Cancer Screening Services. Results from the bivariate and multivariate logistic regression modelling of independent factors associated with utilization of screening services are presented in Table 3. Being above 60 years of age was associated with a 21 times $(\mathrm{AOR}=21.46 ; 95 \% \mathrm{CI}: 6.23,73.93)$ higher odd of utilizing a screening method in this population. Participants who had a monthly income above 305 US Dollars were almost 16 times $(\mathrm{AOR}=15.68 ; 95 \% \mathrm{CI}: 4.60,53.48)$ more likely to report utilization of screening services for prostate cancer than those with lower income. Being knowledgable about prostate cancer and prostate cancer screening methods (AOR $=67.72 ; 95 \% \mathrm{CI}: 8.20,559.57)$ and perceived risk for the development of prostate cancer $(\mathrm{AOR}=16.34 ; 95 \% \mathrm{CI}$ : $7.82,214.92$ ) were also independently associated with higher odds of utilizing screening services among participants in this study (Table 3).

\section{Discussion}

This study aimed to estimate the level of knowledge about prostate cancer and prostate cancer screening services, perceived risk of prostate cancer, utilization of prostate cancer screening services, and associated factors, among men aged 40 years and above in Dar es Salaam, Tanzania.

Our findings indicate that half of the men in this population (52\%) have poor knowledge about prostate cancer. Similar findings have recently been reported among men in Nigeria, South Africa and black men in the Caribbean, America and Africa [10, 13, 14, 18, 19]. Our study findings also corroborate with similar study conducted in Ghana which support the assertation that poor knowledge about prostate cancer is associated with low utilization of screening services [31]. Despite ongoing efforts to raise prostate cancer awareness and knowledge in many African countries, it is obvious that more needs to be done to ensure that men understand the disease and take appropriate action.

Perceived risk of disease has been associated with intention to seek health services including cancer screening services $[13,15]$. A third of participants in this study $(32 \%)$ perceived themselves to not be at risk of prostate cancer, similar to what has been reported in nearby East African country of Kenya and elsewhere in Africa $[5,20]$. A study on the factors influencing men's decision regarding prostate cancer screening in the United States and Africa reported perceived risk of developing prostate cancer to be an important factor for screening uptake [21]. Utilization of health services has been reported to strongly correlate with good knowledge of disease, perceived risk and knowledge about the disease consequencies [22]. Scaling up ongoing efforts to educate the community will go a long way in improving risk perception hence promoting health seeking behaviours.

Utilization of screening services for prostate cancer was uncommon among participants in this study. Only $8 \%$ of men aged 40 years and above, reported to have ever been screened for prostate cancer. Studies in Nigeria, Kenya and elsewhere in Africa have also found alarmingly low use of screening services for prostate cancer. Low utilization of prostate cancer screening have been associated with poor knowledge about prostate cancer and the screening methods in black populations in America and Africa $[10,15,19,23,24]$. It is well known that low utilization of prostate screening services contributes to late diagnosis and increased mortality and morbidity related to prostate cancer $[1,4,5,25]$. Overscreening has potential to cause harm through overdiagnosis and unnessesary invasive procedures, hence its critical that men should be 


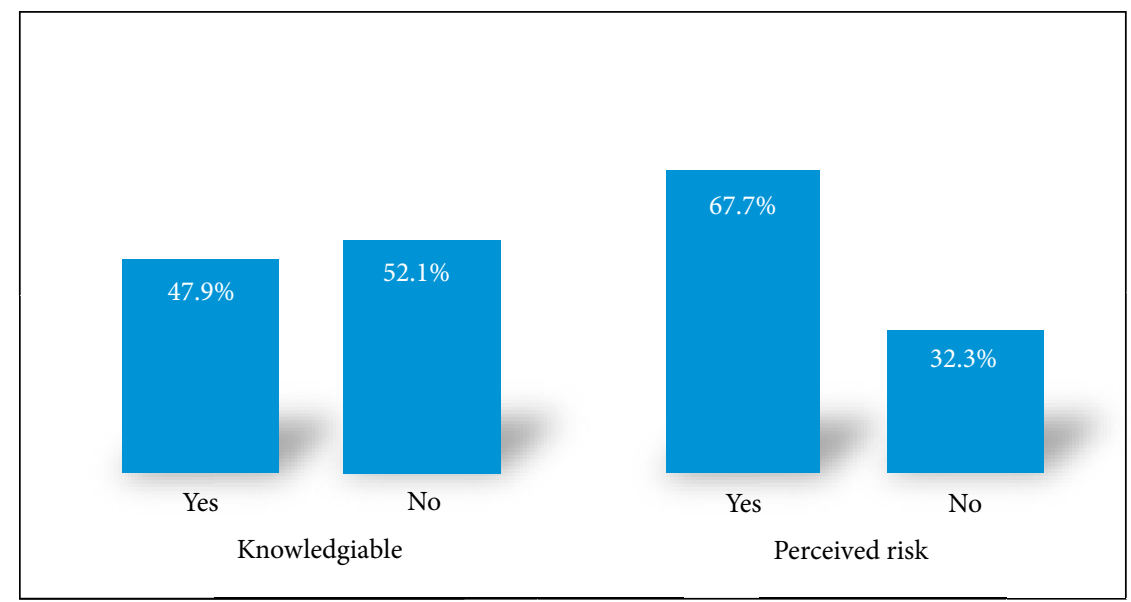

FIGURE 1: Level of knowledge and perceived risk of prostate cancer.

subjected to physical screening based on risk age and having suggestive symptoms. Given that early diagnosis serves life, these findings underscore the importance of efforts geared towards sensitization and knowledge creation among men on the benefits of prostate cancer screening and screening methods in developing countries.

In this study, low utilization of prostate cancer screening services were associated with age, monthly income, and knowledge about prostate cancer and screening services. The association between service utilization and older age may be a result of prolonged exposure to awareness campaigns on prostate cancer but also increased risk and symptoms of prostate cancer with age that may prompt health seeking. Old age has also been associated with decreased sexual activity and increased risk of prostate cancer hence seeking screening services [26]. Similar findings have also been reported elsewhere in Africa, Asia and beyond [3, 8, 15, 23, 27].

Higher income was associated with increased utilization of prostate cancer screening services in this study. Access to health services including screening for cancer has been associated with socio-economic status in several studies worldwide. Education level and income are highly correlated and people who are educated not only have higher odds of better income but also can have access to health education messages, internalize them and act on them. Moreover, income has also been associated with access to health services through increased ability to pay in areas with low coverage of health insurance like Tanzania [28-30]. Though, 48\% of men were found to be aware of the diseases, the utilization of screening was considerably low (8\%). This low utilization of screening services could be explained by low perceived risk, fear of being positively diagnosed, beliefs and cultural barriers towards the screening methods including Digital Rectal Examination (DRE) which is considered to be embarrassing, painful and uncomfortable among men as reported elsewhere in African countries [32-34].

As described earlier, perceived disease risk and knowledge are strong predictors of access to health services. In this study, we found a strong association between the perceived risk of prostate cancer and knowledge of prostate
TABle 2: Participants' knowledge about prostate cancer and screening methods $(n=388)$.

\begin{tabular}{lcc}
\hline Variables & $n$ & $\%$ \\
\hline Know someone who has had prostate cancer before & & \\
Yes & 179 & 46.1 \\
No & 209 & 53.9 \\
Know that prostate cancer is a deadly disease & & \\
Yes & 247 & 63.7 \\
No & 141 & 36.3 \\
Know someone who is suffering from prostate & & \\
cancer today & & \\
Yes & 67 & 17.3 \\
No & 321 & 82.7 \\
Know persons of which sex that are affected by & & \\
prostate cancer & 362 & 93.3 \\
Yes & 26 & 6.7 \\
No & & \\
Know factors that could make a person more likely & & \\
to develop prostate cancer & 153 & 39.4 \\
Yes & 235 & 60.6 \\
No & & \\
Familiar with symptoms of prostate cancer & 106 & 27.3 \\
Yes & 282 & 72.7 \\
No & 199 & 51.3 \\
Prostate cancer curable & 189 & 48.7 \\
Yes & & \\
No & & 62.4 \\
Know prostate cancer screening methods & 37.6 \\
Yes & & \\
No & & \\
\hline & & \\
\hline
\end{tabular}

cancer and screening services with utilization of services. It is therefore of paramount importance to scale up ongoing prostate cancer campaigns among males above 40 years in the country.

The findings presented here should be interpreted in light of a number of limitations. Firstly, the cross sectional nature of the design limits the ability to make causal inference for 
TABLE 3: Univariate and multivariate logistic regression of factors associated with utilization of prostate cancer screening services.

\begin{tabular}{|c|c|c|c|c|c|c|c|c|c|}
\hline \multirow{2}{*}{ Variable } & & \multirow{2}{*}{ Total } & \multirow{2}{*}{$\begin{array}{c}\text { Screened } n \\
(\%)\end{array}$} & \multicolumn{3}{|c|}{ Univariate } & \multicolumn{3}{|c|}{ Multivariate } \\
\hline & & & & $\mathrm{COR}$ & $95 \% \mathrm{CI}$ & $p$-value & AOR & $95 \% \mathrm{CI}$ & $p$-value \\
\hline \multirow{3}{*}{ Age } & $40-50$ & 179 & $5(2.8)$ & 1 & & & 1 & & \\
\hline & $51-60$ & 131 & $5(3.8)$ & 1.38 & $(0.39-4.87)$ & 0.616 & 2.13 & $(0.55-8.22)$ & 0.274 \\
\hline & Above 60 & 78 & $20(25.6)$ & 12.00 & $(4.3-33.41)$ & $<0.001$ & 21.46 & $(6.23-73.93)$ & $<0.001$ \\
\hline \multirow{2}{*}{ Marital status } & Married & 330 & $24(7.3)$ & 0.68 & $(0.27-1.74)$ & 0.422 & & & \\
\hline & Not married & 58 & $6(10.3)$ & 1 & & & & & \\
\hline \multirow{3}{*}{ Education } & $\leq$ Primary & 115 & $6(5.2)$ & 0.61 & $(0.22-1.67)$ & 0.331 & & & \\
\hline & Secondary & 129 & $12(9.3)$ & 1.13 & $(0.49-2.61)$ & 0.778 & & & \\
\hline & $\begin{array}{l}\text { College/ } \\
\text { University }\end{array}$ & 144 & $12(8.3)$ & 1 & & & 1 & & \\
\hline \multirow{2}{*}{ Income } & $>305$ USD & 147 & $26(17.7)$ & 12.52 & $(4.27-36.67)$ & $<0.001$ & 15.68 & $(4.60-53.48)$ & $<0.001$ \\
\hline & $\leq 305$ USD & 237 & $4(1.7)$ & 1 & & & 1 & & \\
\hline \multirow[t]{2}{*}{ Knowledge } & Good & 186 & $29(15.6)$ & 37.13 & $\begin{array}{c}(5.00- \\
275.54)\end{array}$ & $<0.001$ & 67.72 & $\begin{array}{c}(8.20- \\
559.57)\end{array}$ & $<0.001$ \\
\hline & Poor & 202 & $1(0.5)$ & 1 & & & 1 & & \\
\hline \multirow[t]{2}{*}{ Perceived risk } & Yes & 263 & $34(12.9)$ & 13.4 & $\begin{array}{c}(6.12- \\
187.71)\end{array}$ & $<0.001$ & 16.34 & $\begin{array}{c}(7.82- \\
214.92)\end{array}$ & $<0.001$ \\
\hline & No & 125 & $2(1.6)$ & 1 & & & 1 & & \\
\hline
\end{tabular}

factors identified to be associated with prostate cancer screening. However, most factors described have also been published in other studies utilizing various designs and have consistently been associated with service utilization. Secondly, the sample size was calculated to estimate awareness of the screening method and its power to estimate relatively low rate of use is limited. Therefore our estimates of utilization of prostate cancer screening services should be interpreted with caution; thirdly, screening for prostate cancer involves a sensitive procedure particulary if DRE was used. This may have resulted in underestimation of our proportion of utilization due to desirability bias. Efforts were made to build good rapport during interviews and we believe that the accuracy of our estimates may have been strengthened by this, given that these estimates were similar to estimates from elsewhere in Africa.

\section{Conclusions and Recommendations}

This study revealed that the level of knowledge about prostate cancer and screening services is alarmingly low among men in Dar es Salaam. Utilization of screening services for prostate cancer are rare in this population and associated with poor perceived disease risk, low income, lack of knowledge and younger age. Intervention measures aiming at increasing knowledge about prostate cancer and screening services need to be scaled up alongside ensuring that screening services are freely available.

\section{Data Availability}

The data used to support the findings of this study are included within the article.

\section{Conflicts of Interest}

The authors declare that they have no conflicts of interest.

\section{Acknowledgments}

This study was funded by the School of Public Health and Social Science (SPHSS) of the Muhimbili University of Health and Allied Sciences in Collaboration with Centre for Infectious Disease Control (CDC). FB also received support from the Erasmus + Exchange Program between the University of Oslo, Norway and SPHSS to facilitate the preparation of this manuscript.

\section{References}

[1] J. S. H. Ferlay, F. Bray, D. Forman, C. Mathers, and D. M. Parkin, "Estimates of worldwide burden of cancer in 2008: GLOBOCAN," International Journal of Cancer, vol. 127, no. 12, pp. 2893-2917, 2008.

[2] A. Jemal, F. Bray, D. Forman et al., "Cancer burden in Africa and opportunities for prevention," Cancer, vol. 15, no. 118, pp. 4372-4384, 2012.

[3] R. Siegel, E. Ward, O. Brawley, and A. Jemal, "Cancer statistics, 2011 the impact of eliminating socioeconomic and racial disparities on premature cancer deaths," Cancer Journal for Clinicians, vol. 61, no. 4, pp. 212-236, 2011.

[4] F. Okongo, D. M. Ogwang, B. Liu, and D. Maxwell Parkin, "Cancer incidence in Northern Uganda (2013-2016)," International Journal of Cancer, vol. 144, no. 12, pp. 2985-2991, 2019.

[5] D. Adeloye, R. A. David, A. V. Aderemi et al., "An estimate of the incidence of prostate cancer in Africa: a systematic review and meta-analysis," PLoS One, vol. 11, no. 4, p. e0153496, 2016. 
[6] WHO: United Republic of Tanzania, "Country Cancer Profile," World Health Organization, 2014.

[7] A. Ajape, A. Babata, and O. Abiola, "Knowledge of prostate cancer screening among native African urban population in Nigeria," Nigerian Quarterly Journal of Hospital Medicine, vol. 20, no. 2, pp. 94-96, 2010.

[8] A. K. Echimane, A. A. Ahnoux, I. Adoubi et al., "Cancer incidence in Abidjan, Ivory Coast: first results from the cancer registry, 1995-1997," Cancer, vol. 89, no. 3, pp. 653-663, 2000.

[9] O. Oladimeji, Y. O. Bidemi, J.-A. Y. Olufisayo, and A. O. Sola, "Prostate cancer awareness, knowledge, and screening practices among older men in Oyo state, Nigeria," International Quarterly of Community Health Education, vol. 30, no. 3, pp. 271-286, 2010.

[10] H. Nakandi, M. Kirabo, C. Semugabo et al., "Knowledge attitude and practices of Ugandan men regarding prostate cancer," African Journal of Urology, vol. 19, no. 4, pp. 165-170, 2013.

[11] D. Gunda, I. Kido, S. Kilonzo, I. Nkandala, J. Igenge, and B. Mpondo, "Prevalence and associated factors of incidentally diagnosed prostatic carcinoma among patients who had transurethral prostatectomy in Tanzania: a retrospective study," Ethiopian Journal of Health Sciences, vol. 28, no. 1, pp. 11-18, 2018.

[12] Georgeamoah, D. O. Acheampong, G.-S. Kofi et al., "Knowledge, attitude and perception of prostate cancer among male adults in the kumasi metropolis: a descriptive cross-sectional study," Journal of Urology and Research, vol. 5, no. 2, pp. 1-9, 2018.

[13] N. B. O. Mofolo, O. Kenna, S. Koroma, T. Lebeko, F. M. Claassen, and G. Joubert, "Knowledge of prostate cancer among males attending a urology clinic, a South African study," SpringerPlus, vol. 10, no. 4, p. 67, 2015.

[14] R. W. Ojewola, E. S. Oridota, O. S. Balogun et al., "Knowledge, attitudes and screening practices regarding prostatic diseases among men older than 40 years: a population-based study in Southwest Nigeria," Pan African Medical Journal, vol. 27, p. 151, 2017.

[15] C. B. M. D. Steele, C. Maylahn, R. J. Uhler, and C. T. Baker, "Knowledge, attitudes and screening practices among older men regarding prostate cancer," American Journal of Public Health, vol. 90, no. 10, pp. 1595-1600, 2000.

[16] S. B. K. Kanungo, T. Mahapatra, S. Mahapatra, U. K. Bhadra, and K. Sarkar, "Perceived morbidity, healthcare-seeking behavior and their determinants in a poor-resource setting: observation from India," PLoS One, vol. 10, no. 5, p. e0125865, 2015.

[17] Prostate Cancer Awareness, "Knowledge, perception on self-vulnerability and uptake of screening among men in Nairobi County, Kenya," http://ir-library.ku.ac.ke/ handle/123456789/9005.

[18] S. Ikuerowo and S. Ogundele, "Survey of the awareness of prostate cancer and its screening among men attending the outpatient clinics of a tertiary health center in Lagos, Nigeria," Nigerian Journal of Surgery, vol. 21, no. 2, pp. 115-118, 2015.

[19] F. A. Kabore, T. Kambou, B. Zango, and A. Quédraogo, "Knowledge and awareness of prostate cancer among the general population in Burkina Faso," Journal of Cancer Education, vol. 29, pp. 213-221, 2013.

[20] K. Mutua, Anne M. Pertet, and C. Otieno, "Cultural factors associated with the intent to be screened for prostate cancer among adult men in a rural Kenyan community," BMC Public Health, vol. 17, no. 1, p. 894, 2017.
[21] J. M. Ferrante, E. K. Shaw, and J. G. Scott, "Factors influencing men's decisions regarding prostate cancer screening: a qualitative study," Journal of Community Health, vol. 36, no. 5, pp. 839-844, 2011.

[22] E. J. Mmbaga, G. H. Leyna, K. S. Mnyika, and K.-I. Klepp, "Sexually transmitted infections knowledge and its impact in the practice of risky sexual behaviours and HIV serostatus: results from rural Kilimanjaro, Tanzania," Sexually Transmitted Infections, vol. 84, no. 3, pp. 224-226, 2008.

[23] V. H. Pedersen, J. Armes, and E. Ream, "Perceptions of prostate cancer in black African and black Caribbean men: a systematic review of the literature," Psycho-Oncology, vol. 21, no. 5, pp. 457-468, 2018.

[24] S. P. Weinrich, E. Greiner, C. Reis-Starr, S. Yoon, and M. Weinrich, "Predictors of participation in prostate cancer screening at worksites," Journal of Community Health Nursing, vol. 15, no. 2, pp. 113-129, 1998.

[25] P. W. S. Shelton, S. Weinrich and J. W. Reynolds, "Barriers to prostate cancer screening in African American men," Journal of the National Black Nurses Association (JNBNA), vol. 10, no. 2, pp. 14-28, 1999.

[26] A. F. Kotb, A. Beltagy, A. M. Ismail, and M. M. Hashad, "Sexual activity and the risk of prostate cancer: review article," Italian Archive of Urology and Andrology, vol. 87, no. 3, pp. 214-215, 2015.

[27] H. S. Seo and N. K. Lee, "Predictors of PSA screening among men over 40 years of age who had ever heard about PSA," Korean Journal of Urology, vol. 51, no. 6, pp. 391-397, 2010.

[28] H. Amu, K. S. Dickson, A. Kumi-Kyereme, and E. K. M. Darteh, "Understanding variations in health insurance coverage in Ghana, Kenya, Nigeria, and Tanzania: evidence from demographic and health surveys," PLoS One, vol. 13, no. 8, p. e0201833, 2018.

[29] J. Macha, B. Harris, B. Garshong et al., "Factors influencing the burden of health care financing and the distribution of health care benefits in Ghana, Tanzania and South Africa," Health Policy Plan, vol. 27, no. Suppl 1, pp. i46-i54, 2012.

[30] K. Mati, K. K. Adegoke, A. Michael-Asalu, and H. M. Salihu, "Health insurance coverage and access to skilled birth attendance in Togo," International Journal of Gynecology \& Obstetrics, vol. 141, no. 2, pp. 181-188, 2018.

[31] B. Yeboah-Asiamah, D. Yirenya-Tawiah, D. Baafi, and M. M. Ackumey, "Perceptions and knowledge about prostate cancer and attitudes towards prostate cancer screening among male teachers in the Sunyani Municipality, Ghana," African Journal of Urology, vol. 23, no. 3, pp. 184-191, 2017.

[32] I. T. Forrester-Anderson, "Prostate cancer screening perceptions, knowledge and behaviors among African American men: focus group findings," Journal of Health Care for the Poor and Underserved, vol. 16, no. 4A, pp. 22-30, 2005.

[33] K. Mutua, A. M. Pertet, and C. Otieno, "Cultural factors associated with the intent to be screened for prostate cancer among adult men in a rural Kenyan community," BMC Public Health, vol. 17, no. 1, pp. 1-8, 2017.

[34] V. A. Clarke-Tasker and R. Wade, "What we thought we knew: African American males' perceptions of prostate cancer and screening methods," ABNF Journal, vol. 13, pp. 56-60, 2002. 


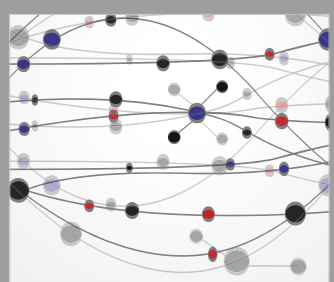

The Scientific World Journal
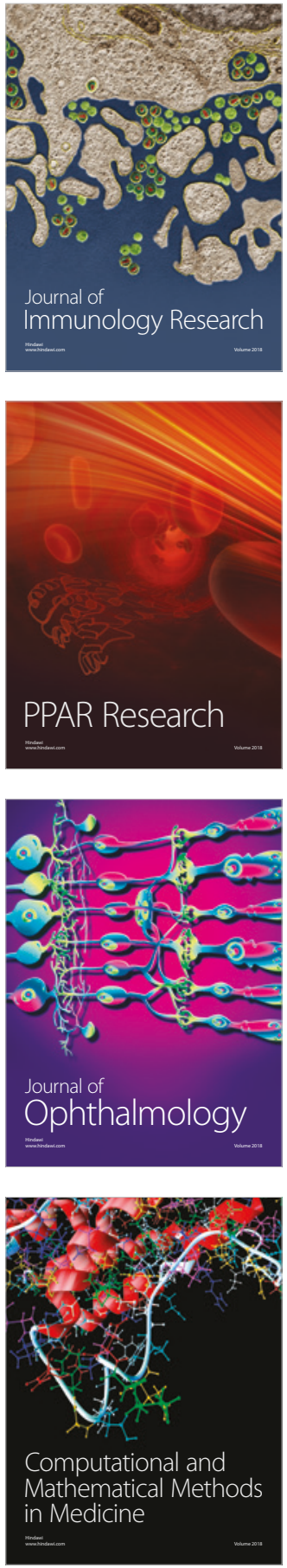

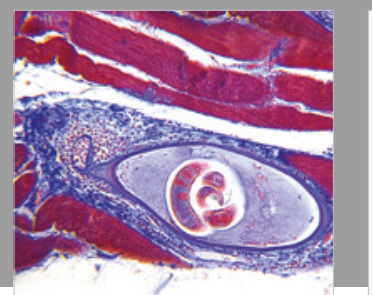

Gastroenterology Research and Practice

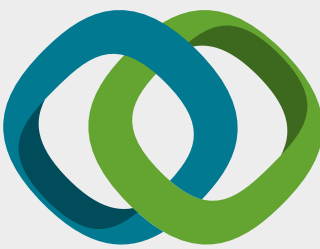

\section{Hindawi}

Submit your manuscripts at

www.hindawi.com
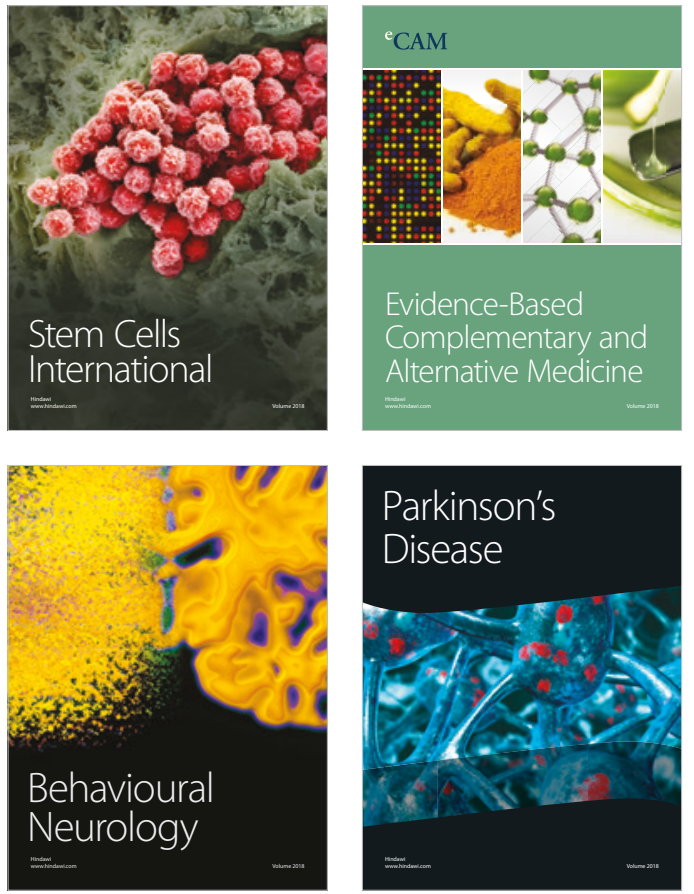

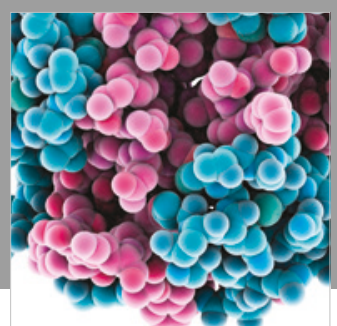

ournal of

Diabetes Research

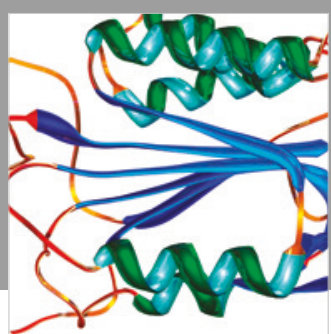

Disease Markers
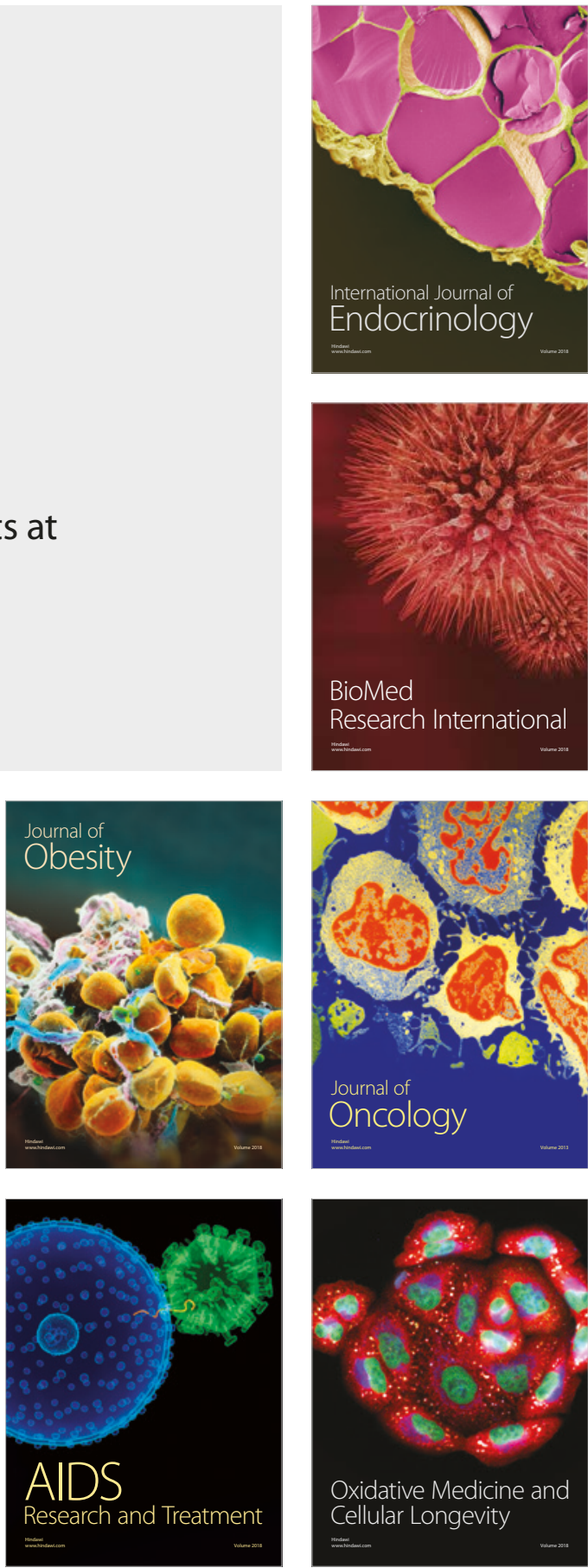